\title{
Revista EDaPECI
}

Grupo de Pesquisa Educação a Distância e Práticas Educativas Comunicacionais e Interculturais Universidade Federal de Sergipe em parceria com Universidade Federal de Alagoas

\section{Conselho Deliberativo}

Prof. Dra. Maria Neide Sobral - Universidade Federal de Sergipe (UFS) - São Cristóvão, Sergipe, Brasil

Prof. Dr. Luís Paulo Leopoldo Mercado - Universidade Federal de Alagoas (UFAL) - Maceió, Alagoas, Brasil

Prof. Dr. Florisvaldo Silva Rocha - Universidade Federal de Sergipe (UFS) - São Cristóvão, Sergipe, Brasil

Prof. Dr. Paulo Heimar Souto - Universidade Federal de Sergipe (UFS) - São Cristóvão, Sergipe, Brasil

\section{Revista EDaPECI}

Grupo de Pesquisa Educação a Distância e Práticas Educativas Comunicacionais e Interculturais

Universidade Federal de Sergipe em parceria com Universidade Federal de Alagoas

\section{Conselho Editorial}

Nacional:

Prof. Dr. Adriano Canabarro Teixeira - Universidade de Passo Fundo (UPF) - Passo Fundo, Rio Grande do Sul, Brasil

Prof. Dr. Agnaldo Arroio - Universidade de São Paulo (USP) - São Paulo, São Paulo, Brasil

Prof. Dr. Antônio Vital Menezes de Souza - Universidade Federal de Sergipe (UFS) - São Cristóvão, Sergipe, Brasil

Prof. Dr. Augusto César Rios Leiro - Universidade Federal da Bahia (UFBA) - Salvador, Bahia, Brasil

Prof. Dr. Cesar Aparecido Nunes - Universidade Estadual de Campinas (UNICAMP) - Campinas, São Paulo, Brasil

Prof. Dr. Edvaldo Souza Couto - Universidade Federal da Bahia (UFBA) - Salvador, Bahia, Brasil

Prof. Dr. Fabiano Ferreira de Castro - Universidade Federal de São Carlos (UFSCar) - São Carlos, São Paulo, Brasil

Prof. Dr. Fábio Alves dos Santos, Universidade Federal de Sergipe (UFS) - São Cristóvão, Sergipe, Brasil

Prof. Dr. Fernando de Almeida Santos - Pontifícia Universidade Católica de São Paulo (PUC-SP) - São Paulo, São Paulo, Brasil

Prof. Dr. Fernando Silvio Cavalcante Pimentel - Universidade Federal de Alagoas (UFAL) - Maceió, Alagoas, Brasil

Prof. Dr. Florisvaldo Silva Rocha - Universidade Federal de Sergipe (UFS) - São Cristóvão, Sergipe, Brasil

Prof. Dr. Dr. Hélio Mário de Araújo, Universidade Federal de Sergipe (UFS) - São Cristóvão, Sergipe, Brasil

Prof. Dr. José Américo Santos Menezes - Universidade Federal de Sergipe (UFS) - São Cristóvão, Sergipe, Brasil

Prof. Dr. José Mário Aleluia Oliveira - Universidade Federal de Sergipe (UFS) - São Cristóvão, Sergipe, Brasil

Prof. Dr. Marco Silva - Universidade do Estado do Rio de Janeiro (UERJ) - Rio de Janeiro, Rio de Janeiro, Brasi

Prof. Dr. Paulo Gileno Cyneiros - Universidade Federal de Pernambuco (UFPE) - Recife, Pernambuco, Brasil

Prof. Dr. Paulo Heimar - Universidade Federal de Sergipe (UFS) - São Cristóvão, Sergipe, Brasil

Prof. Dr. Ronaldo Linhares - Universidade Tiradentes (UNIT) - Aracaju, Sergipe, Brasil

Prof. Dr. Simão Pedro Pinto Marinho - Pontifícia Universidade Católica de Minas Gerais (PUC Minas) - Belo Horizonte, Minas Gerais, Brasil

Profa. Dra. Anamaria Beuno Freitas - Universidade Federal de Sergipe (UFS) - São Cristóvão, Sergipe, Brasil

Profa. Dra. Aneide Oliveira Araújo - Universidade Federal do Rio Grande do Norte (UFRN) - Natal, Rio Grande do Norte, Brasil

Profa. Dra. Anne Alilma Silva Souza Ferrete - Universidade Federal de Sergipe (UFS) - São Cristóvão, Sergipe, Brasil

Profa. Dra. Carla Maria Furuno Rimkus - Universidade Federal de Sergipe (UFS) - Laranjeiras, Sergipe, Brasil

Profa. Dra. Daniela Barros - Fundação Universidade Federal do Rio Grande (FURG) - Rio Grande, Rio Grande do Sul, Brasil

Profa. Dra. Deise Juliana Francisco - Universidade Federal de Alagoas (UFAL) - Maceió, Alagoas, Brasil

Profa. Dra. Divanizia Souza - Universidade Federal de Sergipe (UFS) - São Cristóvão, Sergipe, Brasil

Profa. Dra. Ediméa Oliveira dos Santos - Universidade Federal do Rio de Janeiro (UFRJ) - Rio de Janeiro, Rio de Janeiro, Brasil

Profa. Dra. Eliana Sampaio Romão - Universidade Federal de Sergipe (UFS) - São Cristóvão, Sergipe, Brasil

Profa. Dra. Elizabeth Martines - Universidade Federal de Rondônia (UNIR) - Porto Velho, Rondônia, Brasil

Profa. Dra. Eloiza da Silva Gomes de Oliveira - Universidade do Estado do Rio de Janeiro (UERJ) - Rio de Janeiro, Rio de Janeiro, Brasil

Profa. Dra. Geovana M. Lunardi Mendes - Universidade do Estado de Santa Catarina (UDESC) - Florianópolis, Santa Catarina, Brasil

Profa. Dra. Gicélia Mendes - Universidade Federal de Sergipe (UFS) - São Cristóvão, Sergipe, Brasil

Profa. Dra. Iara Maria Campelo Lima - Universidade Federal de Sergipe (UFS) - São Cristóvão, Sergipe, Brasil

Profa. Dra. Irene Jeanete Lemos Gilberto - Universidade Católica de Santos (UNISANTOS) - Santos, São Paulo, Brasil

Profa. Dra. Isabel Fernandes - Universidade Federal do Rio Grande do Norte (UFRN) - Natal, Rio Grande do Norte, Brasil

Profa. Dra. Lianna Melo Torres, Universidade Federal de Sergipe (UFS) - São Cristóvão, Sergipe, Brasil

Profa. Dra. Lúcia Regina Goulart Vilarinho - Universidade Estácio de Sá (UNESA) - Rio de Janeiro, Rio de Janeiro, Brasil

Profa. Dra. Lucia Santaella - Pontifícia Universidade Católica de São Paulo (PUC-SP) - São Paulo, São Paulo, Brasil

Profa. Dra. Maria Elizabeth Bianconcini de Almeida - Pontifícia Universidade Católica de São Paulo (PUC-SP) - São Paulo, São Paulo, Brasil

Profa. Dra. Maria Helena Silveira Bonilla - Universidade Federal da Bahia (UFBA) - Salvador, Bahia, Brasil

Profa. Dra. Maria Inez Araujo Nascimento - Universidade Federal de Sergipe (UFS) - São Cristóvão, Sergipe, Brasil

Profa. Dra. Maria José Soares Nascimento - Universidade Federal de Sergipe (UFS) - São Cristóvão, Sergipe, Brasil

Profa. Dra. Marizete Lucini - Universidade Federal de Sergipe (UFS) - Itabaiana, Sergipe, Brasil

Profa. Dra. Marlúcia Paiva - Universidade Federal do Rio Grande do Norte (UFRN) - Natal, Rio Grande do Norte, Brasil

Profa. Dra. Marta Fernanda de Araujo Bibiano - Universidade Federal de Pernambuco (UFPE) - Recife, Pernambuco, Brasil

Profa. Dra. Mirella Landim Cysneiros - Universidade Federal de Pernambuco (UFPE) - Recife, Pernambuco, Brasil

Profa. Dra. Nilma Margarida Castro Crusué - Campus de Itapetinga da Universidade Estadual do Sudoeste da Bahia (UESB) - Vitória da Conquista, Bahia, Brasil

Profa. Dra. Patricia Smith - Universidade Federal de Pernambuco (UFPE) - Recife, Pernambuco, Brasil

Profa. Dra. Raquel Colacique - Universidade Federal do Rio de Janeiro (UFRJ) - Rio de Janeiro, Rio de Janeiro, Brasil

Profa. Dra. Samisa Maria Machado (UFS) - São Cristóvão, Sergipe, Brasil

Profa. Dra. Simone de Lucena Ferreira, Universidade Federal de Sergipe (UFS) - Itabaiana, Sergipe, Brasil

Profa. Dra. Vera Wannmacher Pereira, Pontifícia Universidade Católica do Rio Grande do Sul (PUCRS) - Porto Alegre, Rio Grande do Sul, Brasil

Internacional:

Prof. Dr. António Moreira - Universidade de Aveiro (UA) - Aveiro, Portugal

Prof. Dr. Bento Duarte da Silva - Universidade do Minho (UM) - Braga, Portugal

Prof. Dr. Fernando Ramos - Universidade de Aveiro (UA) - Aveiro, Portugal

Prof. Dr. Franscislê Neri de Souza, - Universidade de Aveiro (UA) - Aveiro, Portugal

Prof. Dr. Olívio Patrício - Universidade Técnica de Lisboa (UTL) - Lisboa, Portugal

Profa. Dra. Ana Isabel Mateus da Silva - Escola Superior de Educação de Portalegre (ESEP) - Portalegre, Portugal

Profa. Dra. Branca Miranda - Universidade Aberta (UAb) - Lisboa, Portugal

Profa. Dra. Daniela Barros Universidade Aberta (UAb) - Lisboa, Portugal

Profa. Dra. Daniela Melare Vieira Barros - Universidade Aberta (UAb) - Lisboa, Portugal

Profa. Dra. Lina Morgado - Universidade Aberta (UAb) - Lisboa, Portugal

Profa. Dra. Lúcia Maria Teixeira Pombo - Universidade de Aveiro (UA) - Aveiro, Portugal

Profa. Dra. Maria da Conceição Pereira Ramos - Universidade do Porto (UP) - Porto, Portugal

Profa. Dra. Maria Ivone Clemente Gaspar - Universidade Aberta (UAb) - Lisboa, Portugal

Profa. Dra. Maria José Loureiro - Universidade de Aveio - Lisboa, Portugal

Profa. Dra. Monica Luisa Mendes Baptista - Universidade de Lisboa (ULisboa) - Lisboa, Portugal

Profa. Dra. Natália Ramos - Universidade Aberta (UAb) - Lisboa, Portugal 
EXPEDIENTE

Editores-Gerentes

Fábio Alves dos Santos

Luís Paulo Leopoldo Mercado

Editora Executiva

Rosimeri Ferraz Sabino

\section{Equipe Técnica}

Revisão de Língua Portuguesa

Dalila Santos Bispo

Flávia Ferreira da Silva

Kelly Carine dos Santos

Maria Oscilene de Souza Fonseca
Revisão de Língua Inglesa

Maria Oscilene de Souza Fonseca

Projeto Gráfico

Ozéas Péricles Silva Damasceno
Revisão de Língua Espanhola

Lívia Carvalho Santos

Editor de Layout

Nycolas Menezes Melo

Periodicidade

Quadrimestral

Universidade Federal de Sergipe (UFS)

Reitor: Ângelo Roberto Antoniolli

Vice-Reitor: Iara Maria Campelo Lima

Centro de Educação Superior a Distância (CESAD)

Diretor: Antônio Ponciano Bezerra

Centro de Educação e Ciências Humanas (CECH)

Diretora: Ana Maria Leal Cardoso

Vice-Diretor: Genesio Jose Dos Santos

Departamento de Educação (DED)

Chefe: Sonia Meire Santos Azevedo De Jesus revistaedapeci@gmail.com

Endereço Eletrônico:

http://www.seer.ufs.br/index.php/edapeci

Endereço

Departamento de Educação da UFS

Universidade Federal de Sergipe

Cidade Universitária Prof. José Aloísio de Campos

Av. Marechal Rondon s/n, Bairro Jardim Rosa Elze

São Cristóvão/SE. CEP 49.100-000

Tel. (0xx79) 3194-6757

Data da publicação: dezembro de 2017

\section{Grupo de Pesquisa Educação a Distância e Práticas Educativas Comunicacionais e Interculturais (EDaPECI)}

Líderes do Grupo: Florisvaldo Silva Rocha

Paulo Heimar Souto

Qualquer parte desta publicação pode ser reproduzida, desde que citada a fonte.

Revista EDaPECl- Educação a Distância e Práticas Educativas Comunicacionais e Interculturais / Universidade Federal de Sergipe, São Cristóvão (SE).

Revista EDaPECl, São Cristóvão (SE), v.17. n. 3, p.1-171, set./dez. 2017.

Quadrimestral

ISSN: $2176-171 X$

\section{Indexação}

Sistema Regional de Información en Línea para Revistas Científicas de América Latina, el Caribe, España y Portugal- LATINIDEX; Índice de Revistas de Educación Superior e Investigación Educativa- IRESIE;

Red Iberoamericana de Innovación y Conocimiento Científico- REDIB;

Sistema Eletrônico de Editoração de Revistas- SEER / Instituto Brasileiro de Informação em Ciência e Tecnologia- IBICIT; Sumários de Revistas Brasileiras- Sumarios.org;

Google Acadêmico

Avaliada pelo Qualis/CAPES- Quadriênio 2013-2016:

B1- Ensino, B2- Interdisciplinar, B4- Educação 